\title{
Research Paper \\ Investigation of the Functional Balance in Children With Sensory Impairment Aged 6 to 14 Years Old
}

\author{
Hassan Daneshmandi ${ }^{1}$ (), ${ }^{*}$ Chiman Alyarnezhad ${ }^{1}$ (1)
}

1. Department of Sport Injuries and Corrective Exercises, Faculty of Physical Education and Sports Sciences, University of Guilan, Rasht, Iran.

Citation: Daneshmandi H, Alyarnezhad Ch. [Investigation of the Functional Balance in Children With Sensory Impairment Aged 6 to 14 Years Old (Persian)]. Journal of Sport Biomechanics. 2020; 6(1):22-31. https://doi.org/10.32598/biomechanics.6.1.4

dol $h$ https://doi.org/10.32598/biomechanics.6.1.4

Key words:

Functional balance, Sensory impairment, Hearing impairment, Vision impairment

\section{ABSTRACT}

Objective The balance of human body is maintained by a complex process involving visual, vestibular, and proprioceptive systems. Since the postural control require information from all of these three sensory systems to maintain optimal postural stability, lack of information in one system may affect body sway during maintaining balance. The purpose of this study is to investigate the functional balance in boys with severe-to-profound vision and hearing impairments aged 6-14 years.

Methods This descriptive analytical study was conducted on 30 visually-impaired, 30 hearing-impaired and 30 healthy boys who were selected by purposive sampling method. The Timed Up and Go Test was used for functional balance assessment. One-way ANOVA and Scheffe's post hoc test were used to analyze data in SPSS V. 20 software, considering the significance level at $P \leq 0.05$

Results Functional balance was significantly different in visually-impaired, hearing-impaired and healthy boys $(P=0001)$ where it was poorer in visually-impaired boys compared to visually-impaired and healthy groups $(P=0.000)$. There was no significant difference between hearing-impaired and healthy boys $(P=0.001)$.

Conclusion It seems that vision and hearing impairments can reduce functional balance in boys. Visuallyimpaired boys have poorer balance than the hearing-impaired and healthy boys.

\section{Extended Abstract}

\section{Introduction}

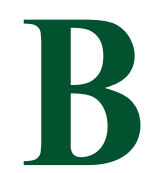

alance is one of the most important and inseparable components of daily activities and exercises [1]. Effective balance is closely related to the ability to perceive the environment through the relevant sensory systems [1]. Sensory systems are proprioceptive, vestibular and visual systems [5]. These systems play an important role in controlling movement in humans [6]. Deficiency of any of this information can affect the balance of systems and lead to problems in balance, posture and coordination
[2]. Sensory impairment is one of the most common causes of functional disability that affects mobility and daily living activities and may be associated with primary and secondary complications [8]. A number of studies have shown that balance is impaired when the ability to receive visual and auditory stimuli is reduced due to visual and auditory impairment or lack of visual and vestibular feedback [13].

Despite the high prevalence of people with sensory impairment in Iran, there is still no accurate information about the postural balance status of this group and the relationship between hearing and vision impairment with the postural balance in visually and hearing impaired people, and few studies have examined the postural balance of these groups of people in a single study. Therefore, it is necessary to con-

\section{* Corresponding Author:}

\section{Chiman Alyarnezhad}

Address: Department of Sport Injuries and Corrective Exercises, Faculty of Physical Education and Sports Sciences, University of Guilan, Rasht, Iran. Tel: +98 (937) 1706087

E-mail: ch.alyarnezhad@yahoo.com 
duct more studies on the functional balance of visually and hearing impaired people, which affects their daily living activities and performance. Since the functional balance test makes it possible to assess the actual movements of individuals, this study examined the functional balance of individuals with visual impairment and hearing loss.

\section{Methods}

This is a descriptive-analytical study. Study population consists of all boys aged 6-14 years with visual impairment and hearing loss. Samples were 30 visually-impaired, 30 hearing-impaired and 30 normal boys (Mean \pm SD age $=11.76 \pm 2.89$ years, Mean \pm SD weight $52 \pm 29.47 \mathrm{~kg}$, Mean \pm SD height $=135.15 \pm 13.13 \mathrm{~cm}$ ) who were non-randomly selected using purposive sampling method. To evaluate the functional balance of subjects, the timed up and go test $(r=0.93)$ was used. In this test, the subject rises from a chair without handle, walks and goes to a chair with handle, sittings on it and then turns around and walks back to the first chair and sits down again. The time of performing this test is considered as the subject's score [15].

Descriptive statistics were used to describe the variables and inferential statistics were used to analyze them. In inferential statistics, after examining the normality of the data distribution by Shapiro-Wilk test, one-way ANOVA and Scheffe's post hoc test were used to compare the three groups at the significance level of $\mathrm{P}<0.05$. in SPSS v. 20 software.

\section{Results}

As shown in Table 1, the results of ANOVA indicated significant difference in the mean functional balance of three study groups $(\mathrm{P}=0.001)$. Scheffe's post hoc test (Table 2) showed that there was a significant difference in functional balance between visually-impaired and hearing-impaired subjects, and between visually-impaired and healthy subjects, but no significant difference was reported between hearing-impaired and healthy subjects.

\section{Conclusion}

The purpose of this study was to evaluate the functional balance of children with visual impairment and hearing loss. The results of this study showed a significant difference in functional balance of two impaired groups compared to controls. This difference was significant between visually-impaired and hearing-impaired children, and between visually-impaired and hearing-impaired children, but no significant difference was observed between hearing-impaired and healthy children. In other words, visually-impaired children had poorer functional balance than healthy and hearing-impaired counterparts which is in line with the results of Farahani et al. and Valizadeh. The results of this study and previous studies in people with visual impairment show that sensory impairment affects the process of functional balance in individuals, such that visual information is more important for postural control than auditory and somatosensorial information.

Table 1. ANOVA results of comparing functional balance in three study groups

\begin{tabular}{cccc}
\hline Group & Mean \pm SD & F & Sig. \\
\hline Visually-impaired & $1.66 \pm 12.49$ & & $0.001^{*}$ \\
Hearing-impaired & $303.47 \pm 0.83$ & 5.87 & \\
\hline Normal & $5.70 \pm 0.99$ & & Journal of \\
\hline Sgnificant at $\mathrm{P}<0.05$. & & Sport Biomechanics
\end{tabular}

Table 2. Pairwise comparison of study groups for functional balance (Scheffe's post hoc test)

\begin{tabular}{cccc}
\hline Group & Mean Difference & Std. Error & Sig. \\
\hline Visually-impaired and hearing-impaired & 0.31 & 6.61 & $0.001^{*}$ \\
Visually-impaired and healthy & 6.78 & 0.31 & $0.001^{*}$ \\
Hearing-impaired and healthy & 0 & 0.31 & 1 \\
\hline
\end{tabular}


Therefore, it can be said that visual information is the richest information in maintaining balance [17]. Investigating the severity of balance disorders that occur in deaf children depends on the flow of information from other systems. This result is consistent with the results of Malekabazdizadeh et al. and Melo et al. It should be noted that in everyday life, vestibular system impairment does not necessarily cause a significant balance disorder due to the overlap of vestibular, visual and somatosensorial inputs and the effect of neural plasticity and the occurrence of central compensation [23]. The results of the present study also show that with the decrease in information receiving from vestibular and visual systems, the balance is impaired, and the person has to use other methods to compensate for this impairment.

\section{Ethical Considerations}

Compliance with ethical guidelines

All subjects voluntarily participated in the present studyafter signing a consent form.

Funding

This research did not receive any specific grant fromfunding agencies in the public, commercial, or not-profit sectors.

Authors' contributions

Conceptualization, methodology, supervision: All authors; Preparing the manuscript, resources: Chiman Alyarnezhad; Review-editing: Hassan daneshmandi.

Conflicts of interest

The authors declared no conflict of interest.

\section{Acknowledgements}

The authors would like to thank the Exceptional Education Organization of Gilan Province, school principals and students who participated in the study for their cooperation. 


\title{
بررسى تعادل عملكردى كودكان داراى اختلال حسى 7 تا ع (ساله
}

\author{
حسن دانشمندى' هـ "جيمن عليارنزاد' هـ \\ 1. كروه آسيبشناسى ورزشى و حركات اصلاحى، دانشكده تربيتبنئى و علوم ورزشى، دانشكاه كيلان، رشت، ايران.
}

\begin{abstract}
حكيد

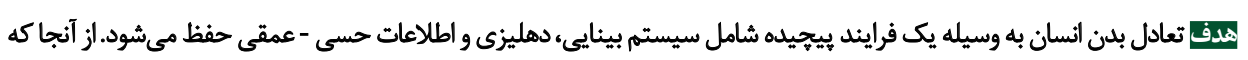

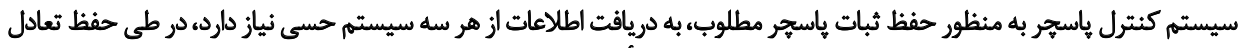

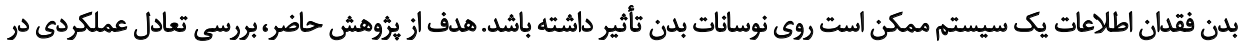

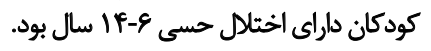

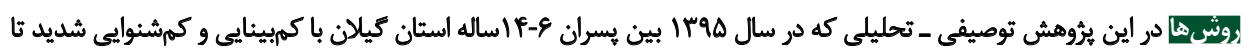

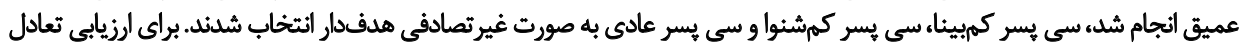

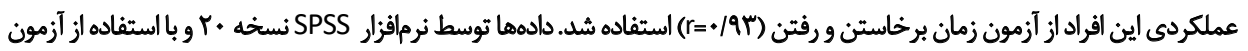

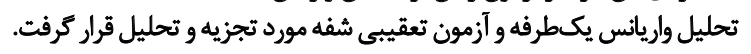

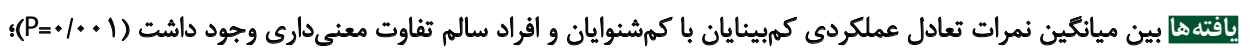

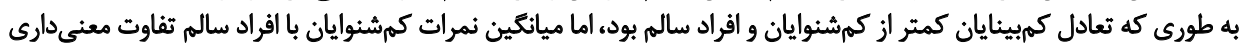

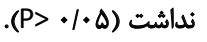

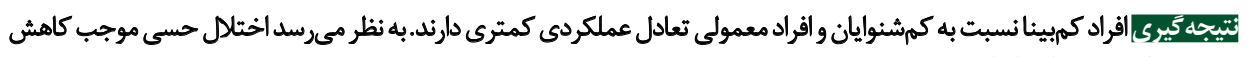
تعادل عملكردى در اين افراد مي شئسود.
\end{abstract}

جاذبه، جهتيابى و حركت سر در فضا و سيستم بينايى براى

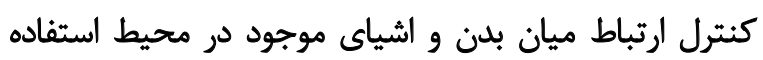

dales

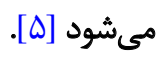

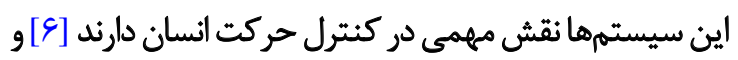

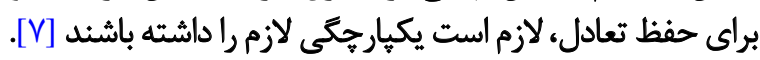

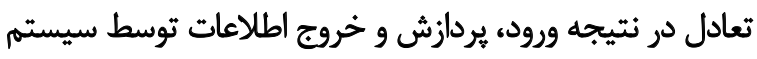

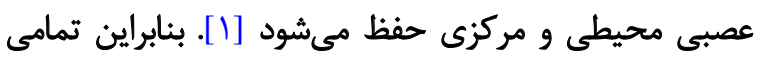

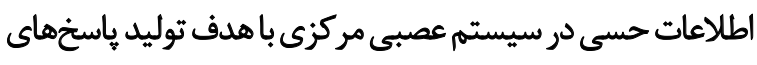

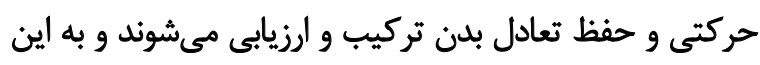

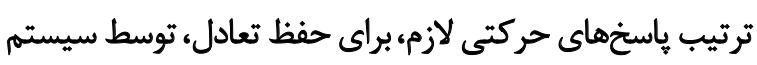

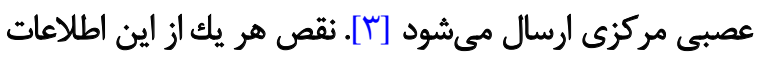

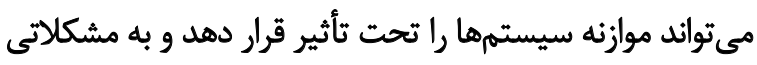

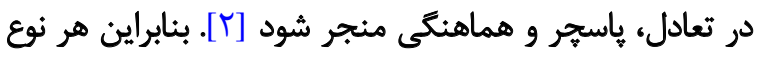

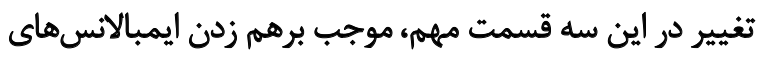

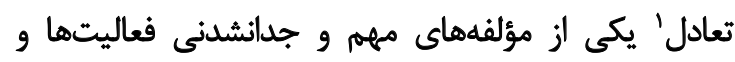

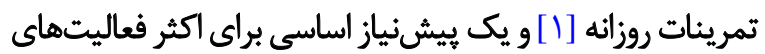

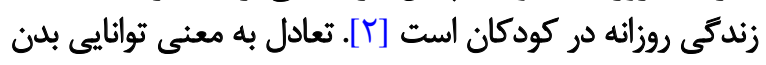

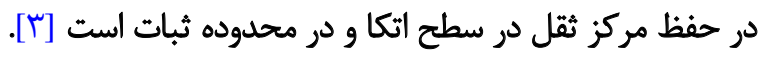

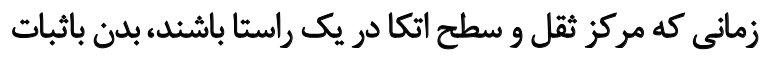

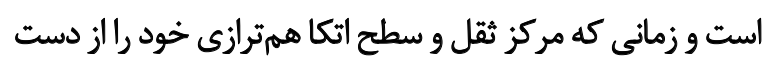

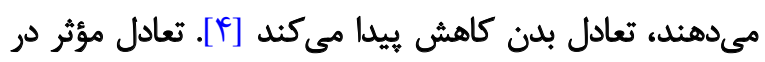

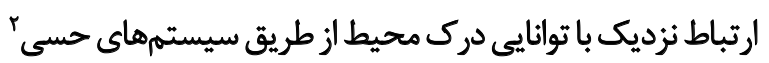

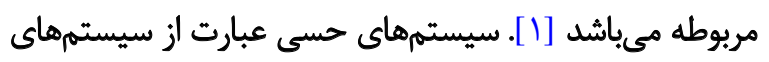

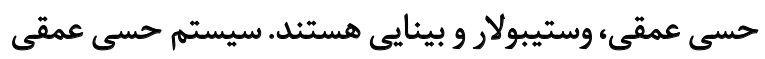
براى كنترل سطح اتكا، سيستم دهليزى برايى كنترل هنئن نيروى

1. Balance

2. Sensory systems 
تعدادى از مطالعات نشان مىدهد زمانى كه امكان دريافت

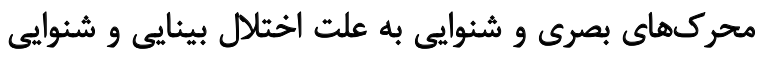

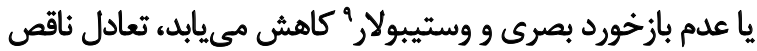

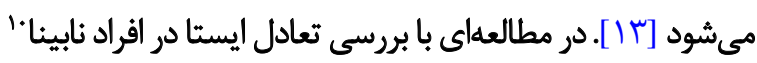

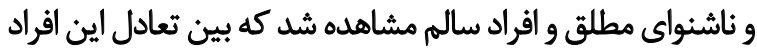

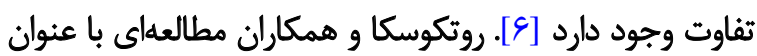

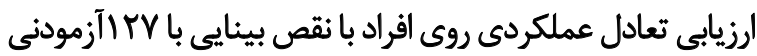

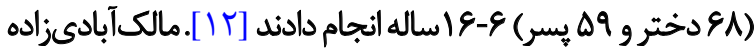

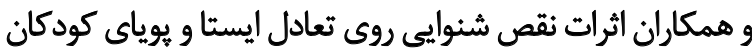

كمشنواى شديد و همتايان سالم را بررسى كردند [1 ]. با بررسى اين مطالعات مشاهده شد اختّلال حسى باعث ضعف

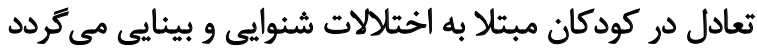

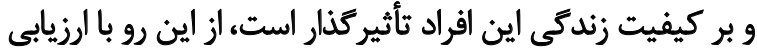

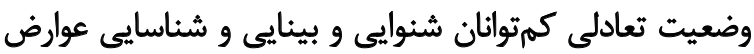

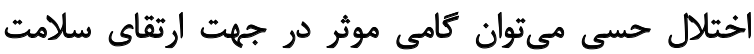
بخش بزركى از جامعه و تضمين زندكى سالمتر و شادابت دابتر آنان

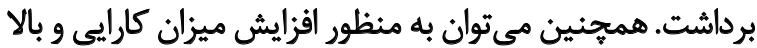

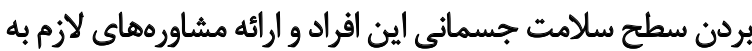

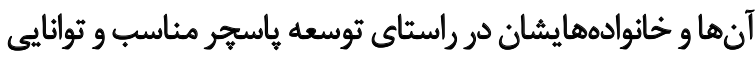
حفظ تعادل بدن تمرينات مناسب راطراحى و برنامهاريزى كردا. باوجود شيوع زياد افراد با اختلال حسى در كشور، هنوز اطلاع

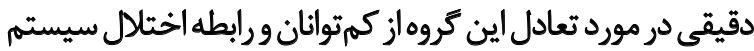

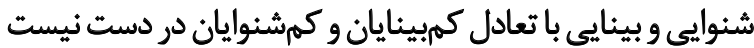

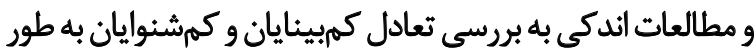

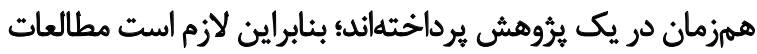

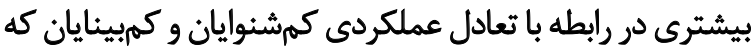

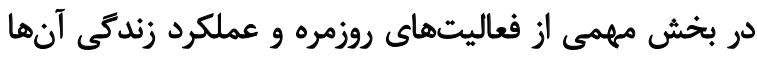

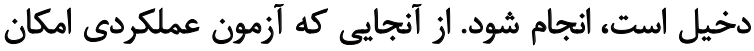

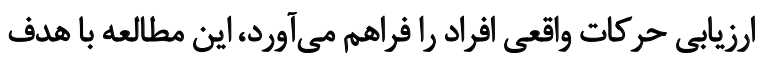
مقايسه تعادل عملكردى كودكان مبتلا به اختلال كمبيناييى،

$$
\text { كمشينوايى و سالم انجام شد. }
$$

در اين مطالعه توصيفى ـ تحليلى از نوع مقايسهاى، جامعه

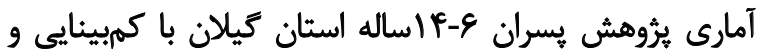

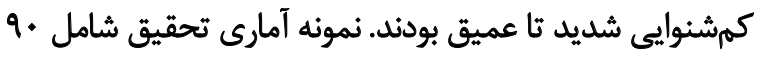

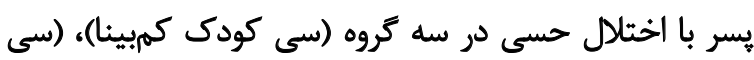
كودى كمشنوا) و (سى كودى عادى) بود، كه در سال هوه بر اساس متغيرهاى سن و جنس بلصورت غيرتصادفى هدف كدار

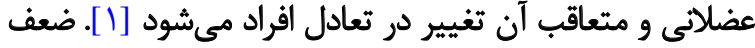

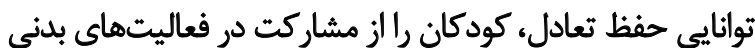

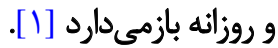

بر اساس تعريف سازمان بهداشت جهانى"، كمثرانى" عبارت است

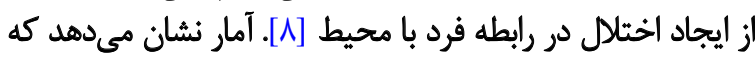

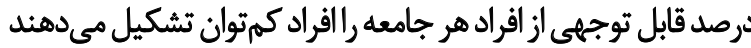

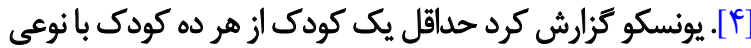

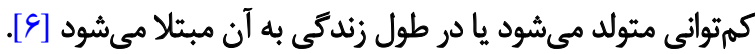

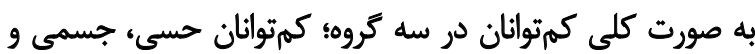

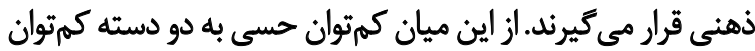

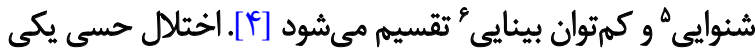

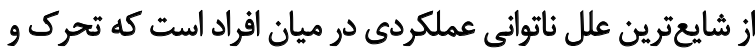

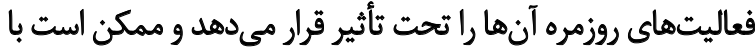

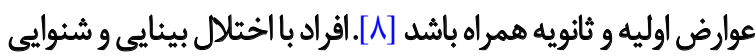
براى جبران آسيبى كه دارند برخى وضعيتهاى نادرست را اتخاذ

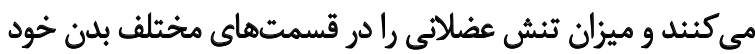

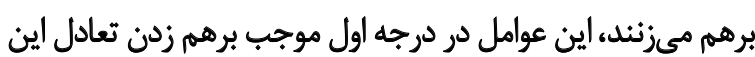

افراد مي بمود [9].

طبق آمارى كه از طرف سازمان بهداشت جهانى منتشر شده است به طور كلى YAD ميليون نفر در سراسر دنيا دجار اختلال

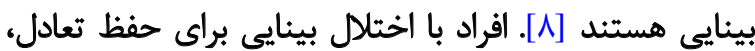

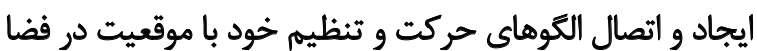
براى جبران عملكرد وايين سيستمهاى بينايى، بايد درخواست

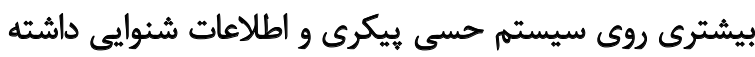

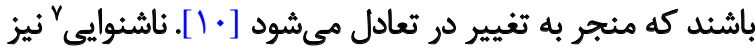

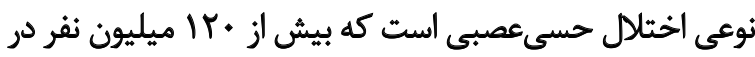

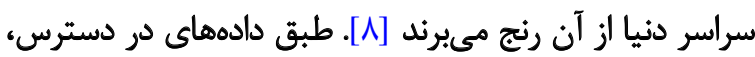

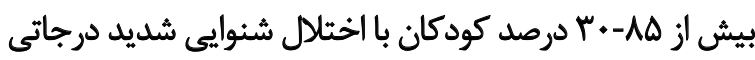

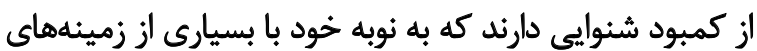

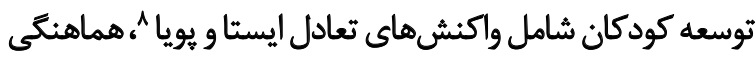
و سرعت انجام حركات تداخل دارد [1 11]. نقص بينايع نهتنهاي بر رشد حس بينايي، بلكه روى همه جنبهائهاى رشد كودى

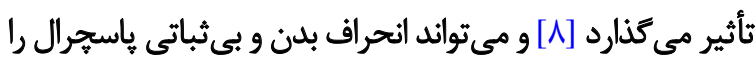

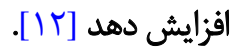

3. World Health Organization (WHO)

4. Disable

5. Hearing impairement

6. Visual impairement

7. Deaf

8. Static and dynamic balance 
و كمشنوايى از بدو تولد بود. با توجه به اينكه جامعه مطالعه

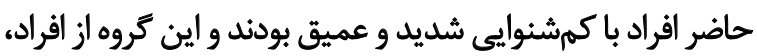

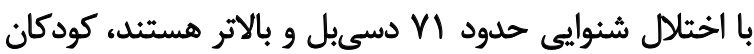
با اين ميزان كمشنوايى براي اين تحقيق در نظر كرفته شداند

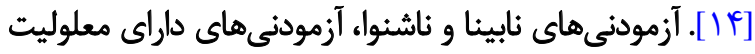

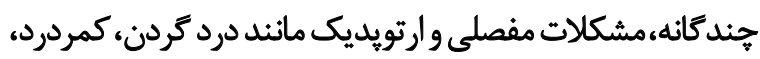

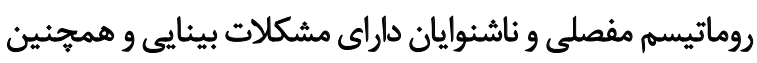

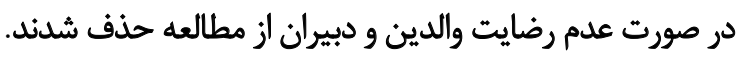
براى ارزيابى تعادل عملكردى اين افراد از آزمون زمان برخاستن

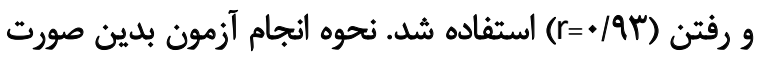

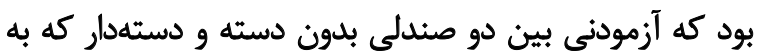

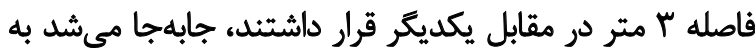

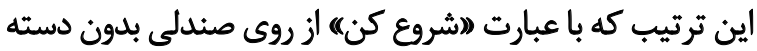

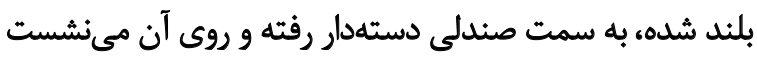

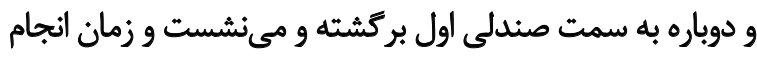

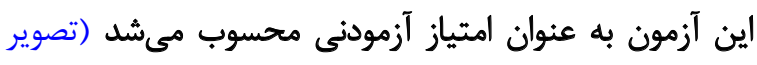

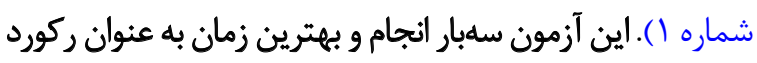

ثبت مىشد [1 1 ].

براى توصيف متغيرها از آمار توصيفى و براي تجزيه و تحليل

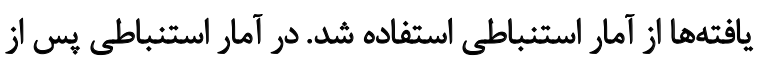
به منظور مقايسه سه كروه از آزمون تحليل واريانس يكسطرانه

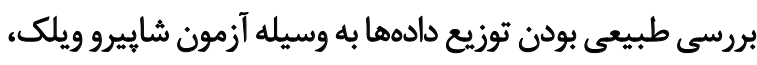

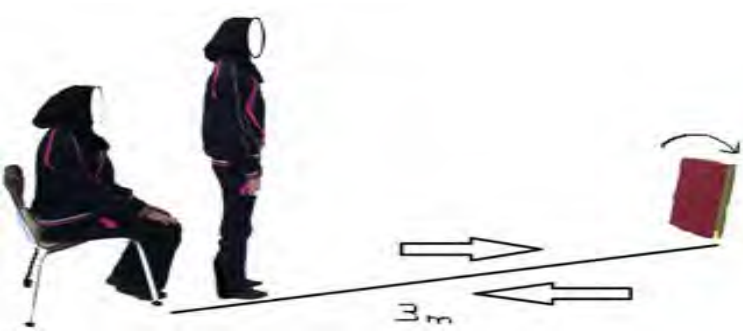

مجله بيومكانيك ونزشـ

$$
\text { تصوير ا. نحوه اندازهيرى آزمون زمان برخاستن و رفتن }
$$

انتخاب شدند و به صورت داوطلبانه در مطالعه شركت كردند. فرم رضايت شركت در تحقيق توسط والدين كودكان مورد مطالعه، تكميل شيد.

بعد از هماهنكي لازم با كروه تحقيقات و يرؤشش آموزش و يرورش

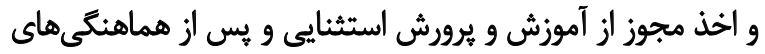

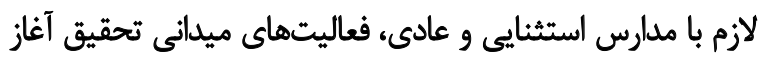

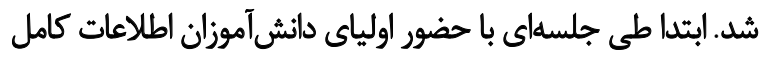

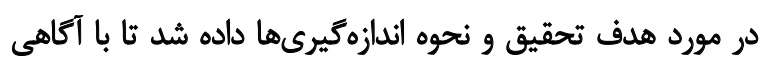

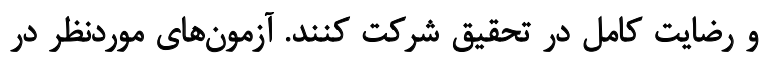
مدارس استثنايى ويروه دانش آموزان با اختالل بينايى و شئوايى انجام

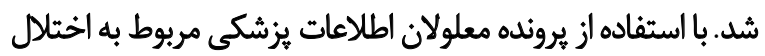
حسى (درجه نقص و سن شروع كمبينايى يا كمشنوايى) ثبت شد.

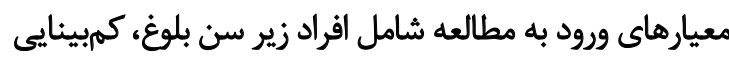

جدول ا. ميائكين و انحراف استاندارد ويرُكى هاى فردى آزمودنىها

\begin{tabular}{|c|c|c|c|}
\hline \multicolumn{3}{|c|}{ ميانكين 土 أنحراف استاندارد } & \multirow{2}{*}{ متغير هاى جمعيتشناختي } \\
\hline سالم & كيششنوا & كهيبنا & \\
\hline $1 r / \varphi+ \pm r / a r$ & $\mid r / \Delta Y \pm T / Q$ & IIVEEY/A9 & سن (سال) \\
\hline $109 / T \cdot \pm 1 Y / A F$ & $\mid Q T / / r \pm I r / N T$ & $|r \Delta / / \Delta \pm| \Psi / N \Psi$ & قد (سانتىمثر) \\
\hline PNAYEINTV & $P F / A E \pm N q F$ & $\Delta T / \cdots \pm Y q / P V$ & ونجن (كيلوكرم) \\
\hline$I V / M \pm r / 19$ & $I V / Y \mp \pm r / I V$ & $19 / 4+ \pm f / 09$ & (كيلو Sرم بر متر مربع) BMI \\
\hline
\end{tabular}

جدول r. نتايج آزمون آناليز واريائ يكطرفه براى مقايسه تعادل عملكردى بين كروههاى كمبيئا، كمشنواو سالم

\begin{tabular}{|c|c|c|c|}
\hline سطح معنيدارى & $\mathbf{F}$ & ميانكين \انحراف استاندارد & كرئو \\
\hline & & $\mid r / e q \pm 1 / F e$ & كمبيينا \\
\hline \multirow[t]{2}{*}{$+1 \cdot+1$} & $r+r / f V$ & $\Delta / A V \pm+/ A \Gamma^{\mu}$ & كمشنوا \\
\hline & & $\Delta / V \cdot \pm \cdot / 99$ & سالم \\
\hline
\end{tabular}


جدول بـ نتايج آزمون تعقيبي شفه براى مقايسه تعادل عملكردى بين كروههاي كيبيئا، كمشئوا و سالم

\begin{tabular}{|c|c|c|c|}
\hline سطح معنى دارى & خطاى استاندارد ميانتين & تفاوت ميانكينها & كروهها \\
\hline$+*+1^{\circ}$ & $+N$ & $9 / 91$ & كمرشنوا -كميينا \\
\hline.$* .1^{\circ}$ & ו & SNM & سالمه -كمبينا \\
\hline 1 & ו &.$/ 19$ & سالم _ كمشنوا \\
\hline
\end{tabular}

در مطالعه خود مشاهده كرد كمبينايان تعادل ايستاي كمترى

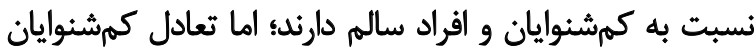

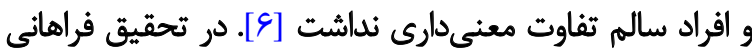

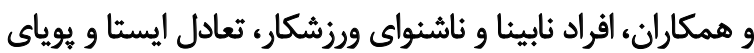

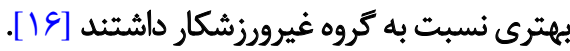
نتايج حاصل از تحليل اين مطالعه و مطالعات بيشين در مورد

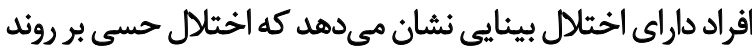

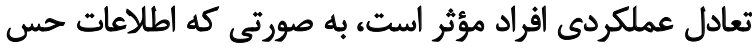

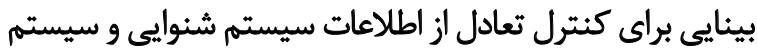
حسى بيكرى مههمتر است. با اين وجود ميتوان كَفت اطنلاعات

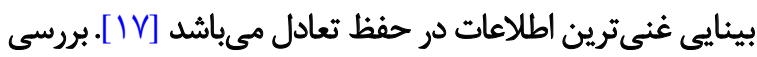

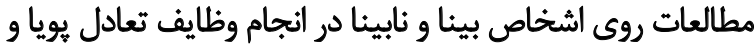

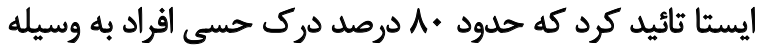
سيستم بينايى كه ديكر ورودىهاى حسى حسى را براى ايجاد تعادل يردازش و كامل مى كند، جمع شده است [N]. در افراد نابينا به دليل حذف بينايى از جمع كل ذخيرهاطلاعات،

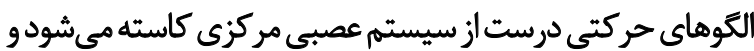

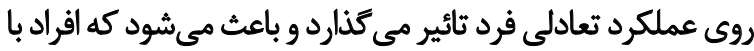

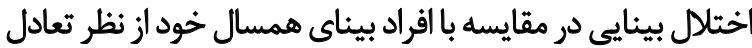

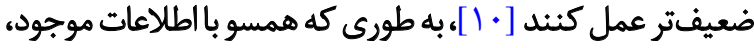

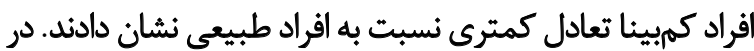

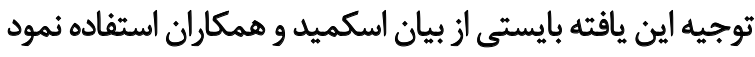

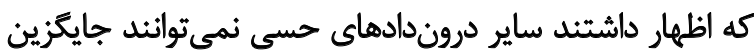

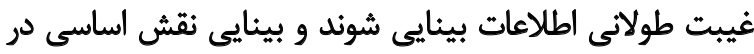

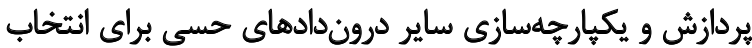

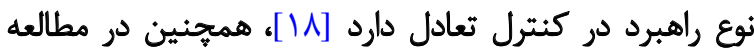

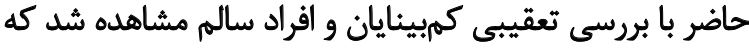

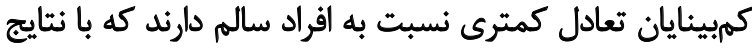

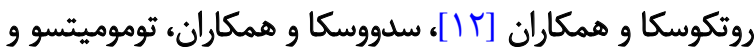

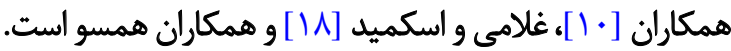

بر اساس نظر شاموى ـ كوى و وولاكوت بحرانىثرين دوره

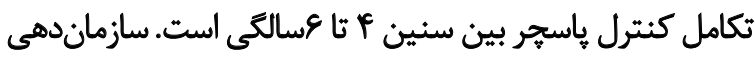
حسى در اين دوره عبارت است أز توانايى سيستم عصبى مركزى
و آزمون تعقيبى شفه در سطح معنى دارى ه.

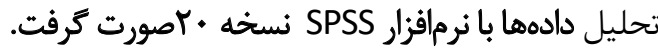

ثنائ

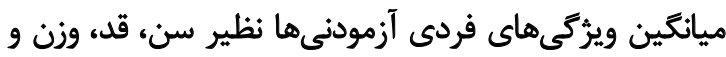

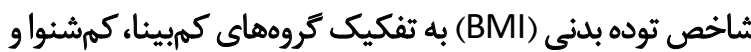
سالم در جدول شماره إ آورده شده است.

بنابر نتايج آزمون آناليز واريانس يكطرفه بين ميانكين نمرات

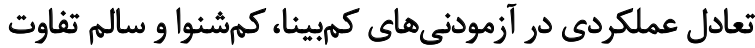

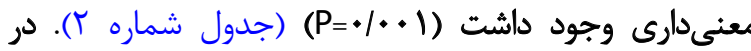

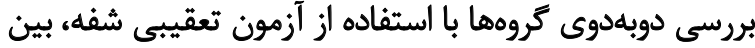

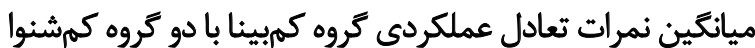

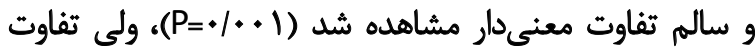

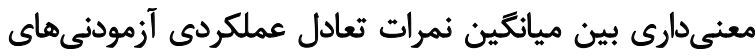

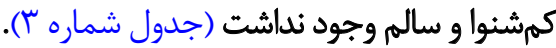

\&s.

كنترل تعادل عملكردى يا به تعريف ديكر توانايى حفظ ثبات

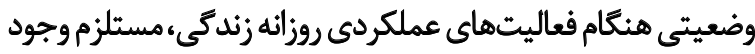

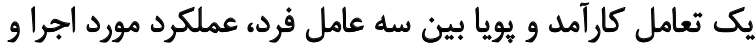

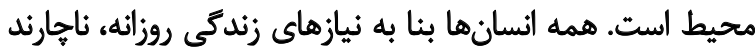

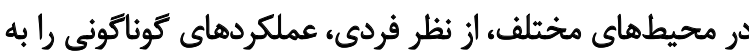

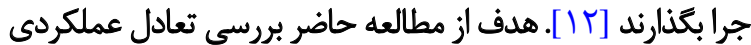
كودكان با اختلال حسى يا كمبينايان و كمشنوايان بود. نتايج اين مطالعه تفاوت معنى دارى در ميزان تعادل عملكردى

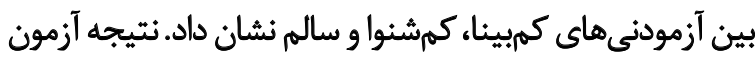
تعقيبى شفه نشان داد كه اين تفاوت بين آزمودنى هاى كمربينا

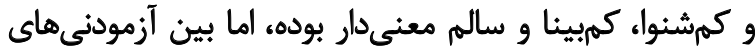

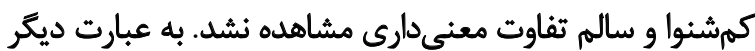
افراد كمبينا نسبت به همتايان سالم و كمشنوا داراى ثعادل

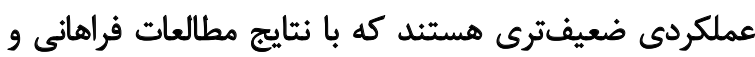
همكاران [19] و ولىزاده و همكاران [9] همسوست. ولىزئزاده 
حس كنترل حركت و جهت كيرى بلن در فضا فراهم مىشود؛؛ براى

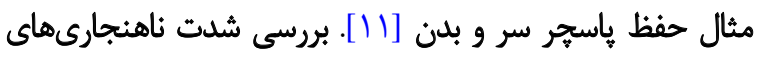

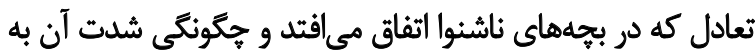

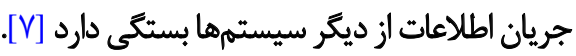

در طرح حاضر با بررسى آزمون تعقيبى شفه كمشنوايان و

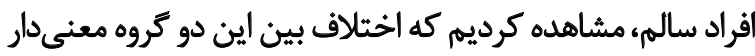

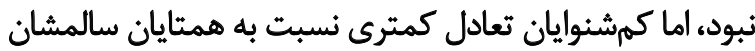

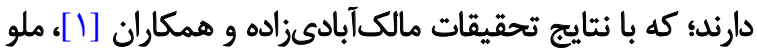

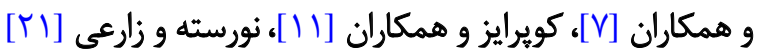

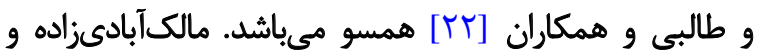

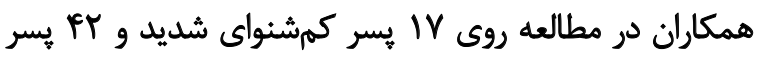

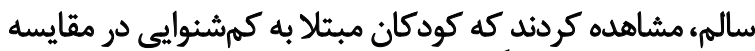

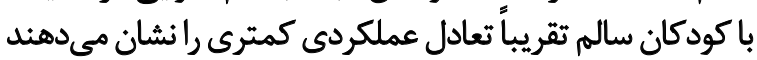

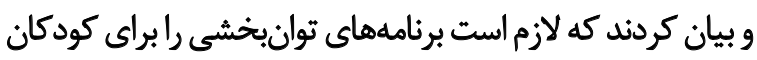

فراهم آورد تا استقلال اصلى اين افراد برقرار شود [ [1 ].

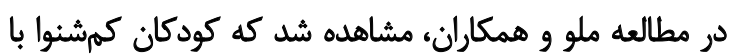

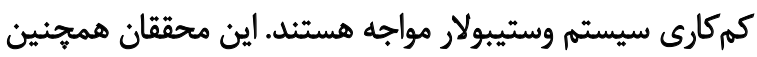
تأكيد كردند كه كم كارى سيستم وستيبولار در كودكان با كمش مشنواييى

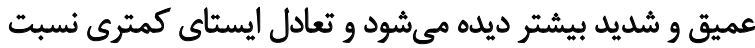

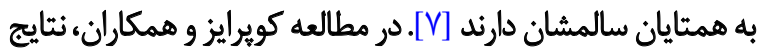

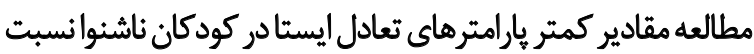

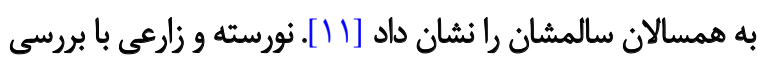
سيستم دهليزى و مهارت حركتى در كودكان با اختلال شنوايى

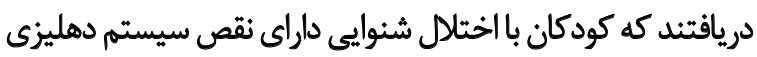
بوده و موقعى مشكل تعادل دارند كه اطلاعات سيستم دهليزى تنها

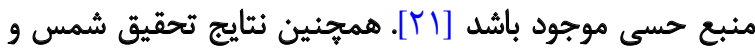
همكاران نشان داد كه آسيب شنوايى خفيف تا متوسط در درئ صورتي

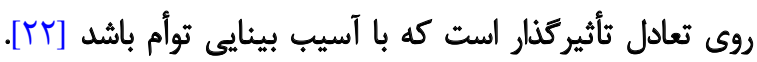

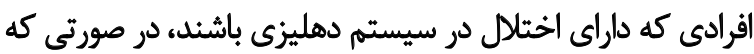

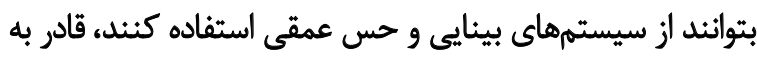

حفظ تعادل خود خواهند بود [•r]

در وضعيتهايى كه دو حس ديكر نيز دجار اختلال شده

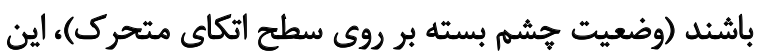

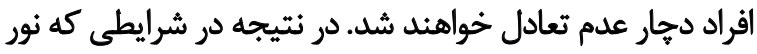

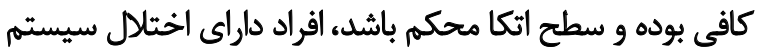

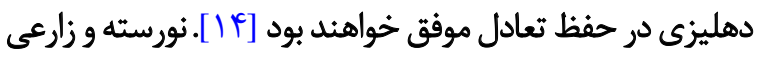

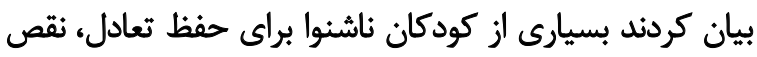

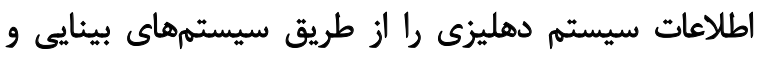
جنبشى جبران مى كنئد [اك]". بايد توجه داشت كه در زندكى روزمره، نقص دهليزى ضرورتاً
در انتخاب، بهكاركيرى و تركيب محركهاى وستيبولار، بينايى

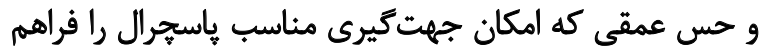

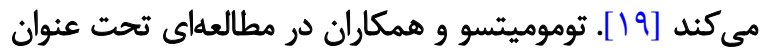

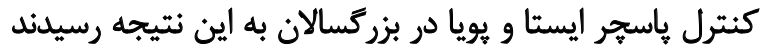

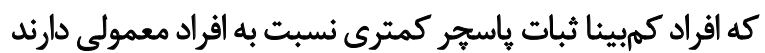

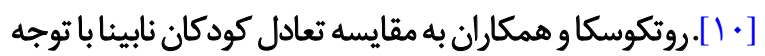

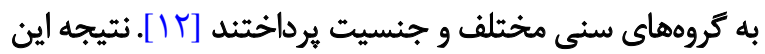

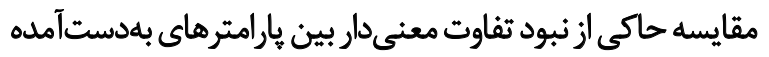

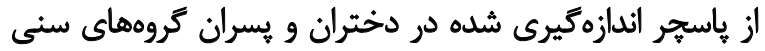

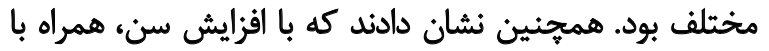

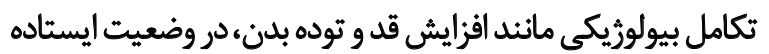

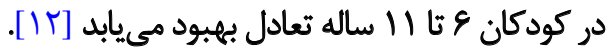

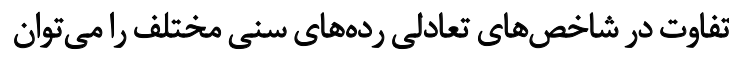

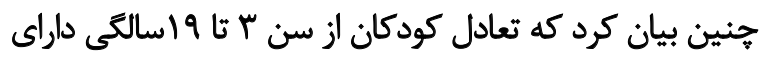

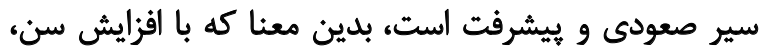

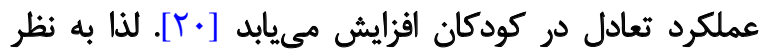

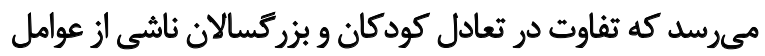

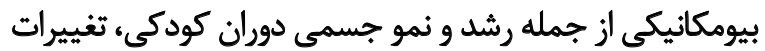

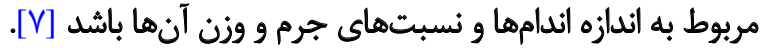

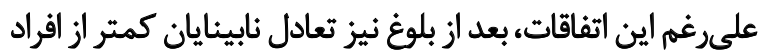

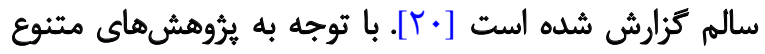

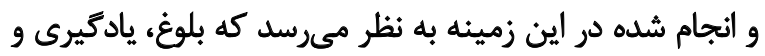

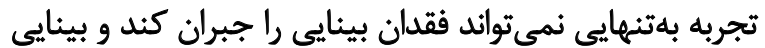

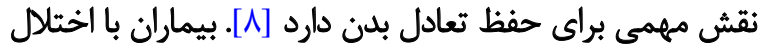

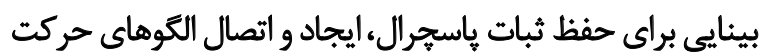

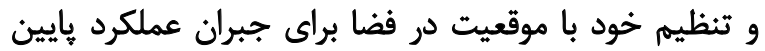

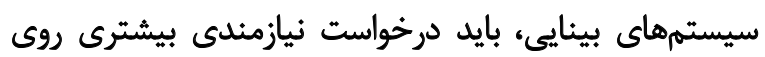

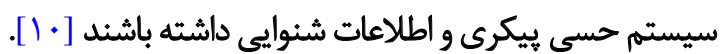
در مطالعاتى بيان شده است كه اطلاعات حسى عمقى در افراد

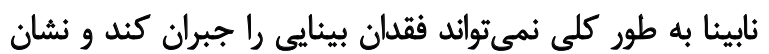

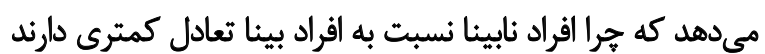

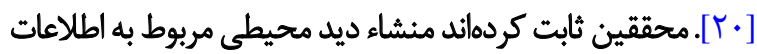

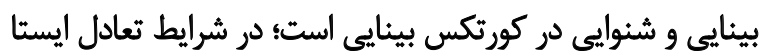

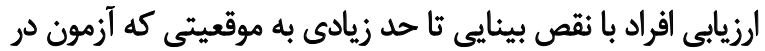

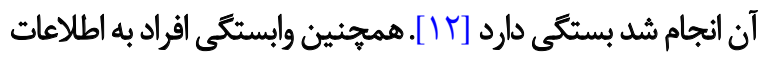

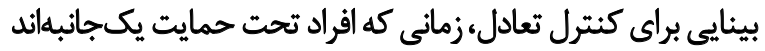

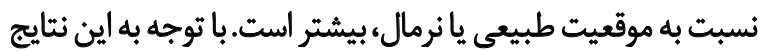

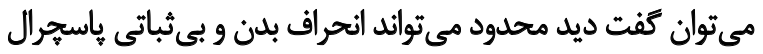

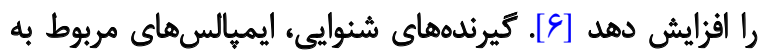

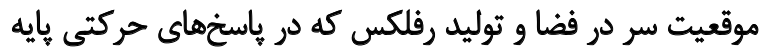

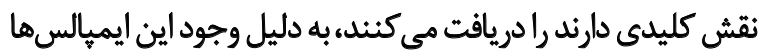


درمجموع آنجه اهميت دارد اين است كه با حفظ تعادل

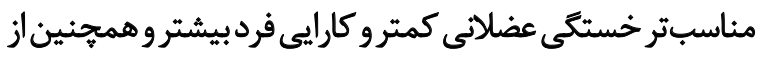
اعمال فشار هاى غيرطبيعى بر قسمتهاي مختلف بد بدن جلوكيري مىشود. بر اين اساس ارزيابي وضعيت تعادلى اين كودكان براي

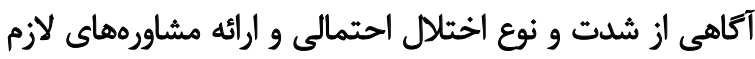

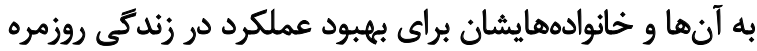
و تجارب محيطى و تفريحى حائز اهميت است. نتايج مطالعه حاضر براي تخصصهاي مختلفى كه با كودكان كمبيينا و كمشنوا

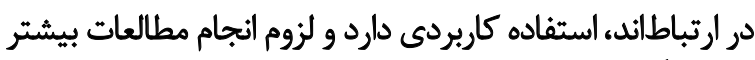

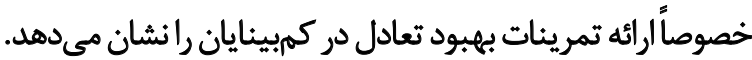

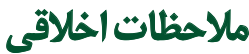

\section{ييروى از اصول اخلاق يثوهش}

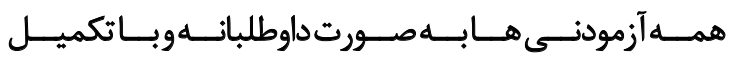

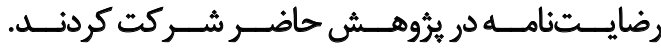

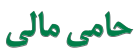

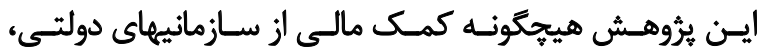

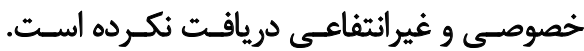

$$
\text { مشاركت نويسند }
$$

مفهومسازى، روش شناسى و نظارت: هر دو نويسندكان؛ بررسى

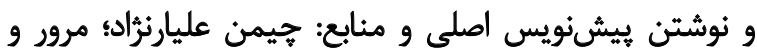
بررسى و ويرايش: حسن دانشمندى.

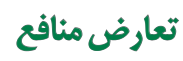

بنابر اظهار نويسندكان اين مقاله تعارض منافع ندارد.

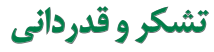

بدينوسيله نويسندكان از سازمان آموزش و برورش استثنايى

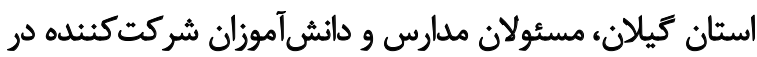
آزمونها كه در انجام تحقيق ما را يارى كردند ماند تشكر و قدر مدردانى

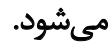

اختلال تعادل بارز و قابل توجهى را باعث نمىشود، كه اين امر

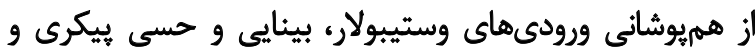

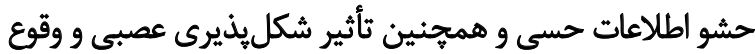

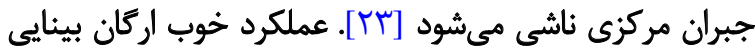

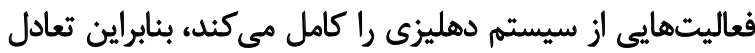

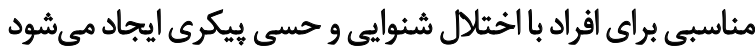

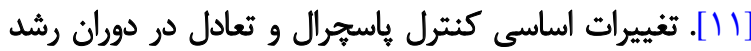
اتفاق مىافتد [ [ ] و كودكان زير سن بلوغ باتغ ممكن است انحراف از

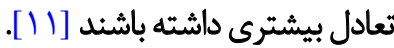

حفظ تعادل در دوران نوجوانى با وجود بينظمى در

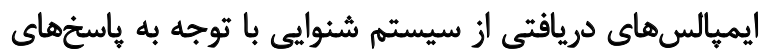

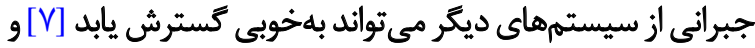

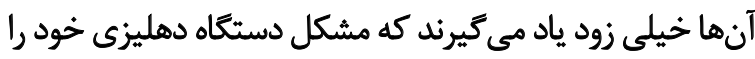

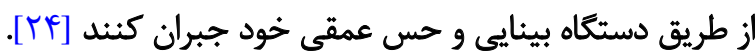

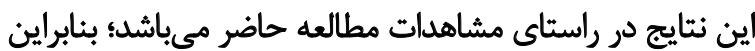

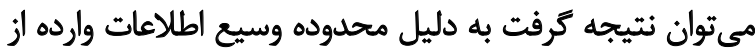

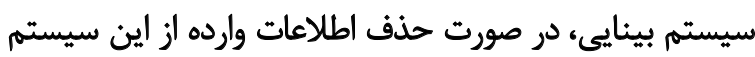
فرد به صورت ناخواسته مجبور است به سيستم شنواييى خود نكيه

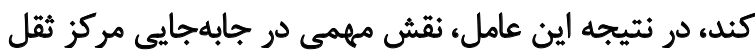

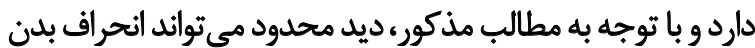

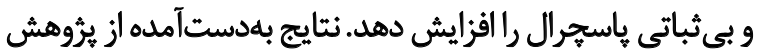

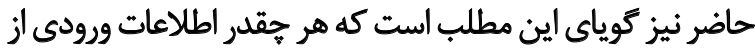

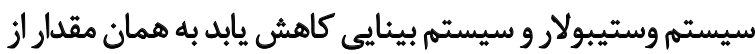

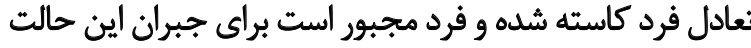

$$
\text { وضعيتهاى جبرانى ديكرى نيز به خود بكيرد. }
$$

\section{نتيجلة تيرىنهايى}

بدن انسان به وسيله يك فرايند يِيجيده شامل اطلاعات بينايي،

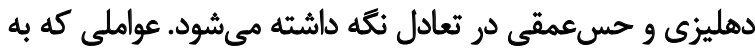

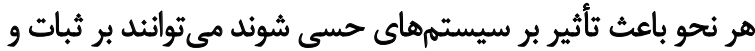

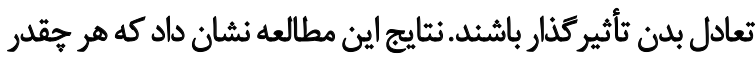

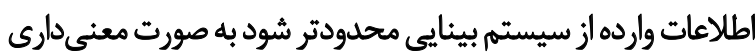

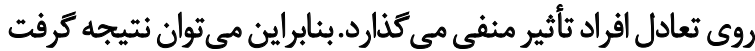

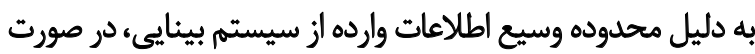

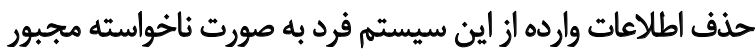

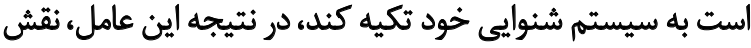

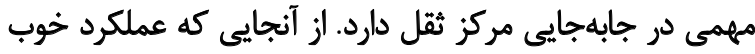

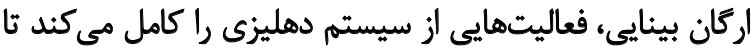

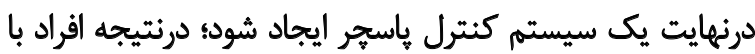
اختلال شنوايي، اختلال تعادل كمثرى رالحساس مي كنيند. 


\section{References}

[1] Malekabadizadeh Z, Barati A, Khorashadizadeh M. The effect of hearing impairment and intellectual disability on children's static and dynamic balance. Aud Vestib Res. 2016; 25(2):82-8. https://avr.tums.ac.ir/index. php/avr/article/view/101

[2] Melo RS, Tavares-Netto AR, Delgado A, Wiesiolek CC, Ferraz KM, Belian RB. Does the practice of sports or recreational activities improve the balance and gait of children and adolescents with sensorineural hearing loss? A systematic review. Gait Posture. 2020; 77:144-55. [DOI:10.1016/j.gaitpost.2020.02.001] [PMID]

[3] de Almeida Ciquinato DS, Doi MY, da Silva RA, de Oliveira MR, de Oliveira Gil AW, de Moraes Marchiori LL. Posturographic Analysis in the Elderly with and without sensorineural Hearing Loss. Int Arch Otorhinolaryngol. 2020; 24(4):e496-e502. [DOI: 10.1055/s-0040-1701271] [PMCID] [PMID]

[4] Daneshmandi H, Majalan AS, Babakhani M, Karanian F. The comparison of head and neck alignment in children with visual and hearing impairments and its relation with anthropometrical dimensions. Phys Ther J. 2014; 4(2):69-76. http://ptj.uswr.ac.ir/article-1-111-en.html

[5] Taheri M, Irandoust KI, Norasteh A, Shaviklo J. [The effect of combined core stability and neuromuscular training on postural control in students with congenital hearing loss (Persian)]. J Res Rehab Sci. 2017; 13(2):80-6. [DOI:10.22122/jrrs.v13i2.2846]

[6] Vali-Zadeh A, Rezazadeh F, A'ali Sh, Mostafa-Zadeh A. [Comparison of static balance among blind, deaf and normal children in different conditions (Persian)]. J Rehab. 2014; 14(4):106-12. http://rehabilitationj.uswr. ac.ir/article-1-1292-en.html

[7] Melo RdS, Lemos A, Macky CFdST, Raposo MCF, Ferraz KM. Postural control assessment in students with normal hearing and sensorineural hearing loss. Braz J Otorhinolaryngol. 2015; 81(4):431-8. [DOI:10.1016/j. bjorl.2014.08.014] [PMID]

[8] Alyarnezhad CH, Danehmandi H, Samami N. [The comparision of upper cross syndrome in children with visual and hearing imoairments with normal counterparts (Persian)]. Res Sport Med Tech. 2018; 16 (15):5765. http://jsmt.khu.ac.ir/article-1-289-en.html

[9] Klavina A, Galeja Z. Relationship between postural alignment in stand ing by photogrametry and static balance in children with disabilities. Proceedings of the International Scientific Conference, Volume III, 2017 May 26-27. Society Integration Education; 2017. [DOI:10.17770/ sie2017vol3.2252]

[10] Tomomitsu MS, Alonso AC, Morimoto E, Bobbio TG, Greve J. Static and dynamic postural control in low-vision and normal-vision adults. Clinics. 2013; 68(4):517-21. [DOI:10.6061/clinics/2013(04)13]

[11] Walicka-Cupryś K, Przygoda $Ł$, Czenczek E, Truszczyńska A, DrzałGrabiec J, Zbigniew T, et al. Balance assessment in hearing-impaired children. Res Dev Disabil. 2014; 35(11):2728-34. [DOI:10.1016/j. ridd.2014.07.008] [PMID]

[12] Rutkowska I, Bednarczuk G, Molik B, Morgulec-Adamowicz N Marszałek J, Kaźmierska-Kowalewska K, et al. Balance functional assessment in people with visual impairment. J Hum Kinet. 2015; 48:99-109. [DOI:10.1515/hukin-2015-0096] [PMID] [PMCID]

[13] Bednarczuk G, Molik B, Morgulec-Adamowicz N, Kosmol A, Wiszomirska I, Rutkowska I, et al. Static balance of visually impaired paralympic goalball players. Int J Sports Sci Coach. 2017; 12(5):611-7. [DOI:10.1177/1747954117727791]
[14] Winnik JP. Adapted physical education and sport. [Daneshmandi $H_{\text {, }}$ Moradi A, Mohammad Ali Nasab E, Karanian F, Yonesi Ramdani A. persian trans]. 1 $^{\text {th }}$ ed. Tehran: Hatmi; 2017.

[15] Alonso AC, Luna NM, Dionísio FN, Speciali DS, Leme LEG, Greve JMDA Functional balance assessment. Medical Express. 2014; 1(6):298-301. [DOI:10.5935/MedicalExpress.2014.06.03]

[16] Farahani R, Noraste AA, Helalat Z, Aghale A. [Comparison of static and dynamic balance Of health men, blind and deaf athletes with nonathletes (Persian)]. Phys Ther J. 2013; 3(1):24-38. http://ptj.uswr.ac.ir/ article-1-66-en.html

[17] Zare S, Rahnama N, Movahedi AR. [The effect of balance exercises on static and dynamic balance of the mentally retarded female students (Persian)]. J Sport Med. 2016; 8(2):143-58. [DOI: 10.22059/JSMED.2017.62303

[18] Schmid M, Nardone A, De Nunzio AM, Schmid M, Schieppati M. Equilibrium during static and dynamic tasks in blind subjects: No evidence of cross-modal plasticity. Brain. 2007; 130(Pt 8):2097-107. [DOI:10.1093/ brain/awm157] [PMID]

[19] Shumway-Cook A, Woollacott MH. Motor control: Translating research into clinical practice. Philadelphia: Lippincott Williams \& Wilkins; 2007. https://books.google.com/books/about/Motor_Control. html?id=BJcL3enz3xMC

[20] A'ali Sh, Rezazade F. [Comparison of physical fitness of blind and deaf children with normal peers (Persian)]. Sport Med Stud. 2015; 5(14):135-50. https://smj.ssrc.ac.ir/article_250_en.html

[21] Norasteh AA, Zarei $H$. [Studying balance in deaf people: A systematic review study (Persian)]. Arch Rehabil. 2019; 20(1):2-15. [In] [DOI:10.32598/rj.20.1.2]

[22] Talebi H, Karimi MT, Abtahi SHR, Fereshtenejad N. Static balance in patients with vestibular impairments: A preliminary study. Scientifica (Cairo). 2016; 2016:6539858. [DOI:10.1155/2016/6539858] [PMID] [PMCID]

[23] Shams A, Aslankhani MA, Abdoli B, Ashayeri H, Namazi Zadeh M. [The effect of visual, proprioception and vestibular systems manipulation on postural control in boys with 4-16 years-old (Persian)]. J Shahrekord Univ Med Sci. 2014; 16(3):22-32. http://journal.skums.ac.ir/article1-1802-en.html

[24] Khodashenas E, Moradi H, Asadi Ghaleni M, Heydari E, Shams A, Enayati $A$, et al. [The effect of selective training program on the static and dynamic balance of Deaf Children (Persian)]. Med J Mashhad Univ Med Sci. 2017; 60(1):383-91. http://mjms.mums.ac.ir/article 9660.html 